\title{
Compression behaviour of hydrate bearing carbonate sand - fines mixtures
}

\author{
$L u \mathrm{MA}^{1,2}$, Abraham C.F. CHIU $^{3^{*}}$ and Y.P. CHENG ${ }^{4}$ \\ ${ }^{1}$ Hohai University, Key Laboratory of Ministry of Education for Geomechanics and Embankment Engineering, Nanjing, China \\ ${ }^{2}$ Anhui Science and Technology University, College of Architecture, Bengbu, China \\ ${ }^{3}$ Shantou University, Guangdong Engineering Center for Structure Safety and Health Monitoring, Shantou, China \\ ${ }^{4}$ University College London, Department of Civil, Environmental and Geomatic Engineering, London, UK
}

\begin{abstract}
High gas hydrate content has been found in fine-grained sediments containing substantial amount of foraminifera in the South China sea. One of the possible hydrate accumulation habits is filling the intraparticle voids in the foraminifera. To understand the effects of this hydrate accumulation habit on the compression behaviour of the fine-grained sediment, two series of isotropic compression tests were conducted. Due to high intra-particle porosity, carbonate sand (CS) was mixed with the fines to mimic the hydrate formation in the intra-particle voids in the laboratory prepared soil specimens. The compression test results revealed that the mixtures of fines with as high as $40 \%$ CS content can exhibit the transitional behaviour such that non-convergent compression lines are observed at the high stress level. It is evident that breakage of CS grains is negligible in these mixtures. Hence, the initial fabrics are not erased under the high stress level resulting in non-convergent compression lines. The compression curves of the hydrate bearing CS-fines mixtures can be classified into three different stress regimes. There is no significant difference in the compressibility of the soil mixtures with and without hydrate in the low stress regime. As the stress increases further reaching the medium stress regime, the hydrate reduces the compressibility of the soil mixtures with increasing hydrate saturation. Upon reaching the high stress regime, the bond breakage at the inter-particle contacts becomes significant leading to the convergence of compression curves between the hydrate bearing and host soil mixtures. It is also found that a new effective hydrate saturation, representing the amount of hydrate in the inter-particle voids, is better correlated to the compressibility of the hydrate bearing soil mixtures.
\end{abstract}

\section{Introduction}

Gas hydrate is considered as one of the potential future energy sources. Past laboratory studies on the mechanical behaviour of hydrate bearing sediments have been mainly focused on coarse-grained soils $[1,2,3]$. The hydrate influences significantly the physical and mechanical properties of the host soils, such as shear wave velocity, compressibility, stiffness and shear strength. Recently there has been an increasing interest in studying the behaviour of hydrate bearing fine-grained soils [4,5]. Past deep sea explorations in the South China Sea have revealed that significant amount of hydrate can be found in the fine-grained sediments containing substantial amount of foraminifera [6]. Foraminifera fossils are shells of carbonate, which are about the size of a sand grain, containing abundant intra-particle pores. It is suggested that the hydrate may form in the intra-particle pores of the foraminifera as well as the inter-particle pores of the sediments. These hydrate accumulation habits are quite different from those commonly found in the coarsegrained soils. Understanding the mechanical behaviour of gas hydrate bearing mixtures of carbonate coarse-grained

\footnotetext{
* Corresponding author: acf_chiu@stu.edu.cn
}

shell and fines is crucial to model properly the performance of geotechnical structures during offshore gas production.

It is well known that the soil structure, a combination of fabric and bonding, play a significant part in the mechanical behaviour of soil. In general sands and reconstituted low plasticity clays exhibit a unique normal compression line because the effects of initial soil structure can be erased at the high stress level, like crushing of sand grains. However, the compression behaviour of the mixtures of coarse-grained and finegrained soils are not well understood. Many gap-graded soils, silts and silty clays exhibit the transitional behaviour such that non-convergent compression lines at the high stress level are observed [7-10]. It is suggested that nonconvergent compression lines are the results of a robust fabric that is unlikely to be erased at the high stress level [11]. However, it is intangible to identify those elements of fabric because it is not easy to recognise the soil fabrics composed of a wide range of grain sizes and different mineralogies [12]. The compression behaviour of the sand-fines mixtures is governed by particle rearrangement or particle crushing mechanism depending on the fines content. In a soil mixtures with high fines content, the 
particle rearrangement becomes the dominant mechanism because crushable sand grains are hard to break [13]. A threshold fines content around $30 \%$ was identified to distinguish between particle arrangement and particle crushing dominant behaviour [14].

In this study the effects of gas hydrate on the compression behaviour of the mixtures of carbonate sand (CS) and fines was investigated. CS was adopted to mimic the foraminifera fossils because both materials exhibit high intra-particle porosity and their major composition is calcium carbonate. Special specimen preparation procedures were used to form the hydrate inside the intraparticle voids of the CS grains. Taking into account the intra-particle porosity, a new parameter, effective hydrate saturation, was defined to interpret the effects of hydrate on the compression behaviour of the soil mixtures.

\section{Laboratory Tests}

\subsection{Materials}

The tested CS was a marine sediment and the fines were crushed quartz taken from a quarry. The basic physical properties of the tested soils are summarised in Table 1. Fig. 1 shows the grain size distribution (gds) curves of the tested soils. CS is classified as a uniform sand and its average grain size $\left(d_{50}\right)$ is $0.75 \mathrm{~mm}$. The grain sizes of fines are smaller than $75 \mu \mathrm{m}$. The size ratio $(R)$ between the coarse-grained CS and the fines is around 17. The CSfines mixtures were formed by mixing $20 \%$ and $40 \%$ CS by weight to the fines. Their gds curves are presented in Fig. 2 (see the solid lines). It is apparent that the two soil mixtures are gap-graded soils.

Table 1. Basic physical properties of tested soils

\begin{tabular}{ccccc}
\hline Soil & $\begin{array}{c}\text { Specific } \\
\text { gravity } \\
\boldsymbol{G}_{\boldsymbol{s}}\end{array}$ & $\begin{array}{c}\text { Grain } \\
\text { size } \\
/ \boldsymbol{m m}\end{array}$ & $\begin{array}{c}\text { Maximum } \\
\text { void ratio } \\
\boldsymbol{e}_{\max }\end{array}$ & $\begin{array}{c}\text { Minimum } \\
\text { void ratio } \\
\boldsymbol{e}_{\min }\end{array}$ \\
\hline $\mathrm{CS}$ & 2.77 & $0.5 \sim 1$ & 1.34 & 0.96 \\
Fines & 2.63 & $<0.075$ & 1.18 & 0.56 \\
\hline
\end{tabular}

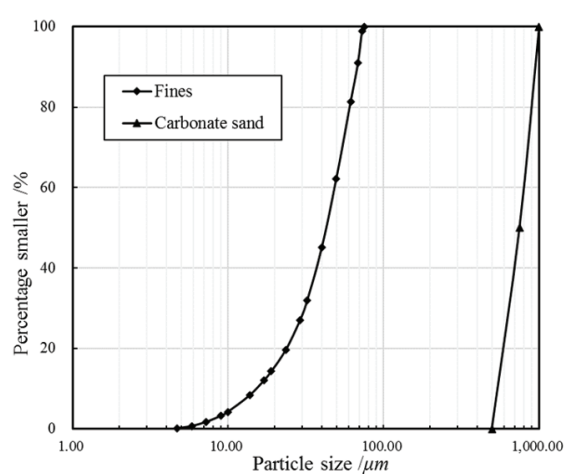

Fig. 1 Grain size distribution curves of carbonate sand (CS) and fines

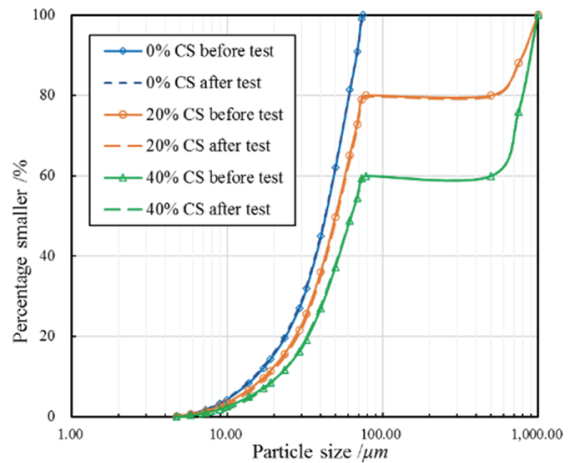

Fig. 2 Grain size distribution curves of CS-fines mixtures before and after compression tests

\subsection{Specimen preparation and compression tests}

Isotropic compression tests were carried out using a temperature-controlled high pressure triaxial test apparatus. Fig. 3 depicts the schematic setup of the testing apparatus. The diameter and height of the soil specimen are $50 \mathrm{~mm}$ and $100 \mathrm{~mm}$, respectively. The test apparatus consists of a high pressure cell chamber equipped with a temperature-controlled system using dimethyl silicone oil, two water pressure controllers, a gas pressure controller and a gas collection system. The system can apply a maximum cell and back pressure of $32 \mathrm{MPa}$ and control a temperature ranging between $-50^{\circ} \mathrm{C}$ and $120^{\circ} \mathrm{C}$.

To mimic the formation of hydrate inside the internal pores of the CS, a given weight of oven-dried CS was first soaked in de-aired water under vacuum to saturate the the internal pores of CS grains. Then a paper towel was used to absorb the water on the surface the CS grains. It should be noted that some water film still found on the surface of CS grains after using the paper towel. The weight of wetted CS was measured carefully to determine the amount of water used in the soil specimen. Thereafter the CS grains were mixed thoroughly with the dry fines. The specimens were compacted to the target dry densities using the moist tamping method. Hydrate was formed inside the specimen using excess gas method. First, the temperature of the specimens were reduced to $-3^{\circ} \mathrm{C}$. Then, methane was injected into the specimen while the cell pressure and gas pressure were increased simultaneously until reaching a gas pressure of $4 \mathrm{MPa}$. An effective stress of $100 \mathrm{kPa}$ was maintained. Then the temperature of cell was increased to $1{ }^{\circ} \mathrm{C}$. Methane hydrate was formed inside the soil specimen under the conditions of $1{ }^{\circ} \mathrm{C}$ and $4 \mathrm{MPa}$. After all the initial pore water reacted with the methane to form the hydrate, de-aired water was allowed to flow from the base pedestal to flush out the excess gas inside the specimen. A back pressure of $8 \mathrm{MPa}$ was applied to saturate the specimen. After saturation, isotropic compression tests were conducted and the specimens were compressed to a maximum pressure of $22 \mathrm{MPa}$. 


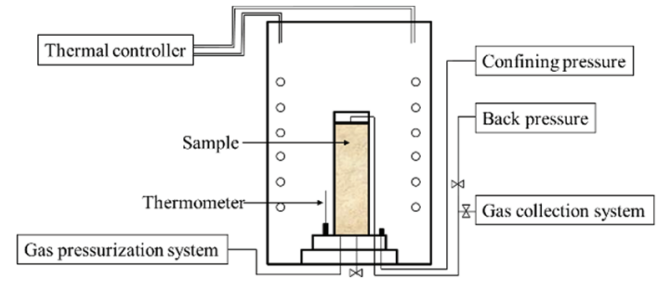

Fig. 3 Schematic setup of temperature-controlled triaxial test apparatus

\section{Results and discussion}

\subsection{Effects of CS content}

Two compression curves of pure fines $\left(\begin{array}{lll}0 \% & \mathrm{CS}\end{array}\right)$ compacted to different initial densities are shown in Fig. 4. The curves exhibit similar trend. A distinct yield stress can be identified, beyond which the compressibilty increases substantially. Unlike clean sands, the compression curves do not converge to a unique compression line despite the specimens were compressed to a high effective stress of $30 \mathrm{MPa}$. The test results are consistent with some fine-grained soils that exhibit the transitional behaviour. In other words, the initial soil fabrics are very robust, which may not be destroyed by the high stress compression. Hence, the initial density is also an important factor governing the compression behaviour of the tested fines.

The effects of CS content on the compression behaviour of the CS-fines mixtures are depicted in Fig. 4. For each soil mixture, two isotropic compression curves of different initial densities are reported. Despite the addition of CS, the compression curves of the CS-fines mixtures follow the trend observed in the pure fines. A distinct yield stress is identified for each compression curve, which increases with increasing initial density for each soil mixture. Besides, no unique compression line is reached under a high effective stress of $30 \mathrm{MPa}$. Hence, all two CS-fines mixtures can be considered as the transitional soil. Past studies on CS have shown that the crushing of sand grains is the principal mechanism leading to a unique compression line. Fig. 2 depicts the gds curves of soil specimens after the compression tests (see the dotted lines). It is apparent that the difference between gds curves before and after the compression tests is not significant.

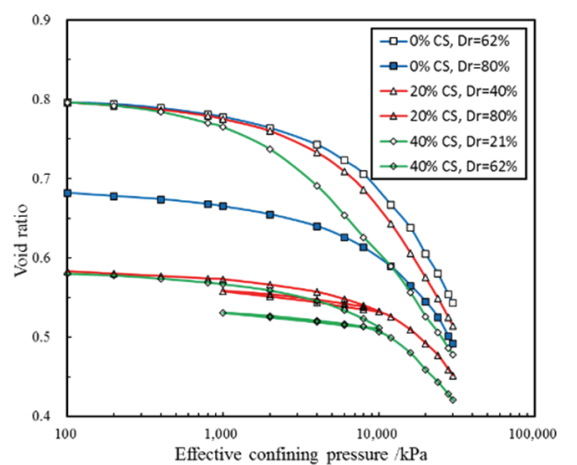

Fig. 4 Isotropic compression curves of CS-fines mixtures

\subsection{Effects of hydrate}

The test procedures adopted in this study could form hydrate in the intra-particle voids of CS and the interparticle voids of soil mixture. Hydrate saturation is used to quantify the amount of hydrate in the specimen. Two saturation parameters are adopted: (i) hydrate saturation, $S_{h}$, is defined as the ratio of the total volume of hydrate $\left(V_{h}\right)$ to the total pore volume of specimen $\left(V_{v}\right)$,

$$
S_{h}=\frac{V_{h}}{V_{v}}
$$

(ii) effective hydrate saturation, $S_{h}$ ', is defined as the ratio of the hydrate volume in inter-particle voids to the volume of inter-particle voids.

$$
S_{h}^{\prime}=\frac{V_{h}-V_{h, c}}{V_{v}-V_{v, c}}
$$

where $V_{h, c}$ is the hydrate volume inside the internal pore of $\mathrm{CS}$ and $V_{v, c}$ is the volume of internal pore of CS.

Figs. 5(a) and $(b)$ show the isotropic compression curves of hydrate bearing CS-fines mixtures. The compression curves are discussed in three different stress regimes. It is apparent that in the low stress regime the compressibility of the hydrate bearing soil mixture is very similar to that of the host soil mixture. For soil mixtures with $20 \% \mathrm{CS}$ and $40 \% \mathrm{CS}, 400 \mathrm{kPa}$ and $1 \mathrm{MPa}$ are the upper limits of the low stress regime, respectively. In the medium stress regime, the effect of hydrate on the compressibility becomes more significant. The compressibility decreases with increasing $S_{\mathrm{h}}$ for the two hydrate bearing soil mixtures. The upper limit of the medium stress regime depends on $S_{\mathrm{h}}$. It is observed that the compression curves of soil mixtures with $S_{\mathrm{h}}>14 \%$ are parallel to those of the host soil mixtures in the pressure ranging between $10 \mathrm{MPa}$ and $25 \mathrm{MPa}$. In other words, the maximum stress applied in the compression tests (22 $\mathrm{MPa}$ ) have not reached the upper limit of the medium stress regime.

On the contrary the compressibility of soil mixtures with $S_{\mathrm{h}}<14 \%$ becomes higher than that of the host soil mixtures in the pressure ranging between $6 \mathrm{MPa}$ and 10 $\mathrm{MPa}$. Beyond the above pressure range, the compression curves of hydrate bearing soil mixtures become closer to those of the host soil mixtures. At the maximum applied pressure of $22 \mathrm{MPa}$, some curves nearly converge to those of the host soil mixtures. Thus, the convergence of compression curves can be observed in the high pressure regime. As the hydrate saturation is low, the bond breakage at the inter-particle contacts may be significant under the applied stress leading to the convergence of compression curves.

In this study the hydrate can form inside the intraparticle voids of CS as well as the inter-particle voids of soil mixture. To distinguish the effects of hydrate formed in the above two different types of voids, the compression curves depicted in Figs. $5(a)$ and $(b)$ are interpreted using $S_{\text {h. }}$. It can be seen from the figures that the compressibility increases substantially beyond an effective confining pressure of $6 \mathrm{MPa}$. Thus, $6 \mathrm{MPa}$ is adopted as a reference pressure to define a void ratio index $\triangle e_{6}$, which is the difference of void ratio on the compression curves between the hydrate bearing and the host soil mixture 
corresponding to this reference pressure. $\triangle e_{6}$ can quantify the compressibility of hydrate bearing specimen with reference to the one without hydrate. The relationships between void ration index $\left(\triangle e_{6}\right)$ and hydration saturation $\left(\mathrm{S}_{\mathrm{h}}\right), \triangle e_{6}$ and effective hydrate saturation $\left(\mathrm{S}_{\mathrm{h}}{ }_{\mathrm{h}}\right)$ for the two soil mixtures are shown in Figs. 6(a) and $(b)$.

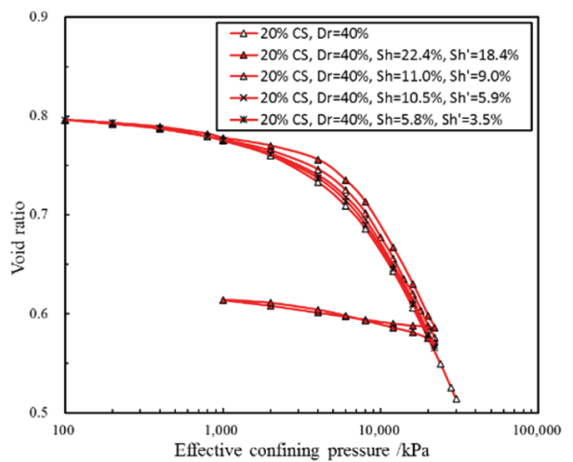

(a)

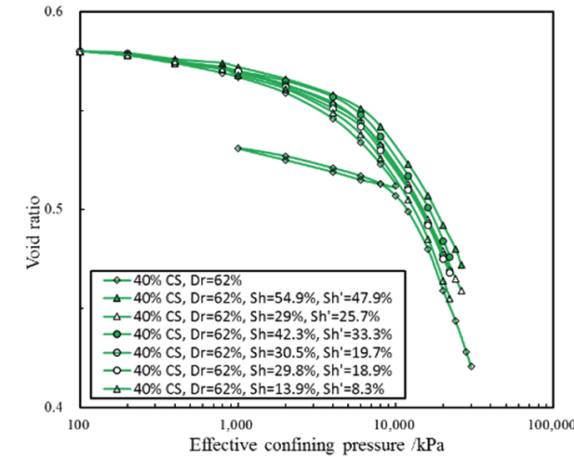

(b)

Fig. 5 Isotropic compression curves of hydrate bearing CSfines mixtures (a) 20\% CS and (b) $40 \% \mathrm{CS}$

Fig. 6(a) shows four specimens of the soil mixture with $20 \%$ CS. It should be noted that the difference between $S_{h}$ and $\mathrm{S}_{\mathrm{h}}$ is the amount of hydrate in the intra-particle voids. The specimens have different proportions of hydrate in the inter-particle and intra-particle voids. The specimens are classified into two groups based on a similar amount of hydrate in the intra-particle voids. $S^{\prime}{ }_{\mathrm{h}}$ of the specimens in the first group are $3.5 \%$ and $9 \%$. The remaining two specimens in the second group have a $S_{\mathrm{h}}$ of $5.9 \%$ and $18.4 \%$. Compared with $\mathrm{S}_{\mathrm{h}}$, a higher coefficient of determination $\left(R^{2}\right)$ is obtained for the correlation between $\triangle e_{6}$ and $S^{\prime}$. Thus, it is suggested that the hydrate formed inside the inter-particle voids plays more important role in enhancing the compressibility of the soil mixture. Fig. $6(b)$ shows six specimens of the soil mixture with $40 \%$ CS. The specimens also have different proportions of hydrate in the inter-particle and intra-particle voids. Similar to Fig. 6(a), the correlation between $\triangle e_{6}$ and $S^{\prime}{ }_{\mathrm{h}}$ has a higher value of $R^{2}$.

Fig. 2 reveals that the crushing of weak CS grains is insignificant for the range of stress applied. Thus the strength enhancing of CS grains due to the filling of hydrate in the intra-particle voids may not be a principal factor governing the compressibility of the soil mixtures. On the contrary, the enhancing effect of hydrate either inside the inter-particle voids or at the inter-particle contacts are more significant.

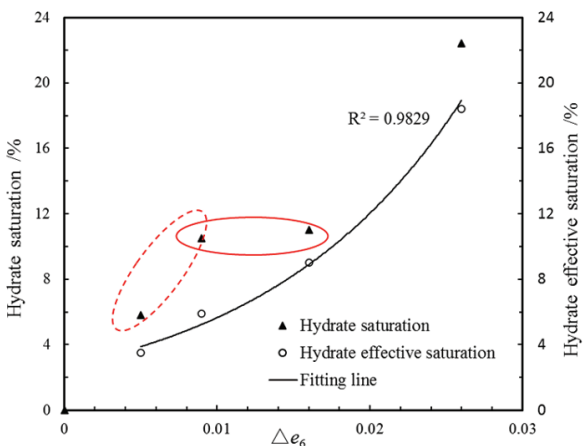

(a)

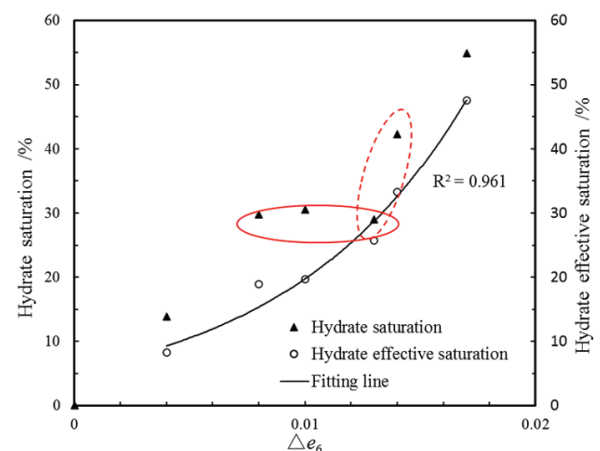

(b)

Fig. 6 Relationships between void ratio index and hydrate saturation and effective hydrate saturation for the hydrate bearing soil mixtures: (a) 20\% CS, and (b) $40 \%$ CS

\section{Conclusions}

This paper presents a laboratory study on the effects of hydrate on the compression behaviour of the carbonate sand (CS)-fines mixtures. The main conclusions are shown as follows:

(1) The quartz fines exhibit the transitional behaviour such that non-convergent compression lines are observed at the high stress level. The mixtures of CS-fines remain the transitional behaviour despite adding CS content up to $40 \%$. It is evident that crushing of the weak CS grains is negligible. Thus, the initial stable soil fabrics do not erase after compression leading to non-convergent compression lines at the high stress level.

(2) Due to the high intra-particle porosity of CS, hydrate can form in its intra-particle voids as well as the inter-particle voids of the soil mixtures. The compression curve of hydrate bearing CS-fines mixture consist of three stress regimes. The stress limits between the stress regimes depends on the hydrate saturation. In the low stress regime, no significant difference in compressibility between the hydrate bearing and the host soil mixture is observed. As the stress increases further and reaches the medium stress regime, the compressibility decreases with increasing hydrate saturation. After reaching the high stress regime, the bond breakage at the interparticle contacts becomes significant. Hence, the compressibility of hydrate bearing soil mixture 
becomes higher than that of the host soil mixture. As the stress increases further, the two compression curves converge to each other.

(3) A void ratio index is proposed to quantify the compressibility of hydrate bearing soil mixtures. An empirical correlation between the void ratio index and the effective hydrate saturation is suggested. It is postulated that the hydrate formed in the inter-particle voids plays a more important role in the compression behaviour of the hydrate bearing soil mixtures.

The authors would like to acknowledge the financial support of Key projects of natural science research in colleges and universities of Anhui Province (KJ2018A0539, KJ2020A0080).

\section{References}

[1] C.R.I. Clayton, J.A. Priest, E.V.L. Rees. The effects of hydrate cement on the stiffness of some sands. Geotechnique 60, 435-45 (2010)

[2] S. Dai, J.C. Santamarina, W.F. Waite, T.J. Kneafsey. Hydrate morphology: Physical properties of sands with patchy hydrate saturation. J. Geophy. Res.: Solid Earth 117, B11205 (2012)

[3] M. Hyodo, J. Yoneda, N. Yoshimoto, Y. Nakata. Mechanical and dissociation properties of methane hydrate-bearing sand in deep seabed. Soils \& Found. 53, 299-314 (2013)

[4] M. Hyodo, Y. Wu, K. Nakashima, S. Kajiyama, Y. Nakata. Influence of fines content on the mechanical behavior of methane hydrate-bearing sediments. J. Geophy. Res.: Solid Earth 122, 2017JB 014154 (2017).

[5] L. Liang, J.C. Santamarina. Laboratory strategies for hydrate formation in fine-grained sediments. J. Geophy. Res.: Solid Earth 123, 2017JB014624 (2018)

[6] X. Wang, D.R. Hutchinson, S. Wu, S. Yang, Y. Guo. Elevated gas hydrate saturation within silt and silty clay sediments in the shenhu area, south china sea. J. Geophy. Res.: Solid Earth 116, B05102 (2011)

[7] F.B. Martins, L.A. Bressani, M.R. Coop, A.V.D. Bica. Some aspects of the compressibility behaviour of a clayey sand. Can. Geotech. J. 38, 1177-1186. (2001)

[8] L. Xu, M.R. Coop. The mechanics of a saturated silty loess with a transitional mode. Geotechnique 67, 581-596 (2017)

[9] B. Shipton, M.R. Coop. Transitional behaviour in sands with plastic and nonplastic fines. Soils \& Found. 55, 1-16 (2015)

[10] M.C. Todisco, M.R. Coop, J.M. Pereira. Fabric characterisation in transitional soils. Granul. Matter 20, 20 (2018)

[11] F. Altuhafi, B.A. Baudet, P. Sammonds. The mechanics of subglacial sediment: an example of new "transitional" behaviour. Can. Geotech. J. 47, $775-790$ (2010)

[12] P.M.V. Ferreira, A.V.D. Bica. Problems in identifying the effects of structure and critical state in a soil with a transitional behaviour. Geotechnique 56, 445-454 (2006)

[13] E. Elkamhawy, B. Zhou, H.B. Wang. Transitional behavior in well-graded soils: An example of completely decomposed granite. Eng. Geol. 253, 240-250 (2019)

[14] T.D. Pitman, P.K. Robertson, D.C. Sego. Influence of fines on the collapse of loose sands. Can. Geotech. J. 31, 728-739 (1994) 